\title{
Las mujeres en la prensa deportiva: dos perfiles
}

\author{
Women in sports newspapers: two patterns
}

\section{Mulheres nos esportes jornais: dois padróes}

\author{
Clara Sainz de Baranda Andújar
}

Profesora Ayudante. Miembro del Instituto Universitario Estudios de Género de la Universidad Carlos III de Madrid

\begin{abstract}
RESUMEN: El propósito del presente estudio fue analizar la imagen que la prensa deportiva espańola transmite de las mujeres y cómo ha evolucionado en los últimos años (1979 - 2010). Para ello, se realizó un análisis de las informaciones donde al menos aparecía una mujer $(\mathrm{n}=4877)$ en los cuatro periódicos deportivos de mayor tirada. Y dentro de las informaciones se analizaron a los protagonistas (hombres o mujeres) $(n=20928)$ y se examinando siete variables para determinar los perfiles de mujer $(\mathrm{n}=12195)$ que protagonizan las informaciones: número de protagonistas por información, género, si es protagonista principal, la ocupación, su función en la información, la relaciones familiares mencionadas, cómo se presenta a esa persona en la información y si está directamente citada en el artículo. En general se distinguen dos perfiles de mujeres que protagonizan la prensa deportiva: la propia del 'Ámbito Deportivo', y “otras” que se han dado en llamar 'Invitadas', por lo general familiares, parejas, famosas o aficionadas. Se observa un aumento de la presencia de estas últimas a lo largo del tiempo lo que produce un aumento de la invisibilidad de las deportistas.

Palabras clave: prensa deportiva, perfiles de mujer, medios de comunicación, deportistas

Abstract: The purpose of this study was to find out which image of women transmits Spanish sports newspapers and how it has evolved in recent years (1979-2010). Were analyzed all articles where a woman appeared $(\mathrm{n}=4877)$ in the four largest circulation sports newspapers. And then, were analyzed the protagonists (men or women) $(n=20928)$ and seven items to determine the patterns of women $(n=12195)$ on the sport news: main character amount, gender, if main character, occupation (or charge), its role in the information, mentioned family relationships, how that person presents in-
\end{abstract}

formation and if it is directly cited in the article. The results show that there are two patterns of women in the Spanish sport news: the characteristic 'Sportswomen' and "other", that we have called "invited", usually they are families, couples, famous or lovers. We observed an increase in the presence of the "invited" woman over time, and this is one of de causes which increase invisibility of 'Sportswomen'.

Key words: sports newspapers, patterns of women, mass media, sportswomen

Resumo: O objetivo deste estudo foi descobrir qual imagem da mulher transmite jornais desportivos espanhóis e como ela tem evoluído nos últimos anos (1979-2010). Foram analisados todos os artigos em que uma mulher apareceu $(\mathrm{n}=4877)$ nos quatro maiores jornais desportivos de circulação. E, em seguida, foram analisados os protagonistas (homens ou mulheres) $(\mathrm{n}=20928)$ e sete itens para determinar os padróes de mulheres $(\mathrm{n}=$ 12.195) sobre a notícia do esporte: quantidade personagem principal, sexo, o personagem protagonista, ocupação (ou carga ), o seu papel na informaçấo, indicado relaçôes familiares, como pessoa que apresenta informaçôes e se é directamente citado no artigo. Os resultados mostram que existem dois padróes de mulheres nas notícias do esporte espanhol: a característica "Atletas" e "outro", que temos chamado de "convidados", geralmente são famílias, casais, famoso ou amantes. Observou-se um aumento na presença da mulher "convidados" ao longo do tempo, e esta é uma das causas de que aumentam a invisibilidade dos "Atletas".

Palavras chave: jornais desportivos, padróes de mulheres, meios de comunicação, desportistas

\section{Introducción}

La información deportiva tiene una gran demanda en la sociedad actual, prueba de ello es el tiempo que invierten los noticiarios e informativos en dar cuenta de la actualidad deportiva o el hecho de que Marca sea el diario de pago más leído en España, con casi tres millones de lectores al día (Estudio General de Medios (EGM), 2012). Los medios de comunicación constituyen una ineludible fuente de información e influencia para la ciudadanía (Duncan, 1990; Lumpkin \& Williams, 1991) lo que hace necesario revisar cómo

Dirección para correspondencia [Correspodence address]: Dpto de Periodismo y Comunicación Audiovisual (despacho 17.02.01). Universidad Carlos III de Madrid. C/Madrid 126, 28903 Getafe (Madrid). Telf: 916248552 / 6492892 38. E-mail: cbaranda@hum. uc3m.es está influyendo el discurso en la sociedad española actual, en la labor de promoción deportiva entre la población de cualquier edad, sexo y condición.

El género como categoría de análisis remite a una noción de relación: relación de la mujer con su entorno, pero también como contraposición al hombre. Por lo tanto, se impone la necesidad de abordar el papel de las deportistas pero también el de los deportistas para entender la realidad que los medios de comunicación transmiten del deporte en general (Ramajo y Lallana, 2011, p. 181-183).

Los diferentes estudios sobre el tratamiento de la información deportiva denuncian la trivialización de los contenidos, un aumento de los estereotipos y la escasa representación de mujeres deportistas (Rintala y Birrell, 1984; Fink y Kensicki, 2002; Sainz de Baranda, 2013). En cualquier caso, aunque el 
análisis englobe a medios generalistas, las mujeres están en clara desventaja respecto a los hombres, más aún cuando nos centramos en el caso de España.

En EE.UU. en los años noventa se produce un aumento de la información deportiva femenina, fundamentalmente tras los éxitos de las atletas estadounidenses en los Juegos olímpicos de 1996. Sin embargo, autores como Kane y Greendorfer (1994, p. 40) ya anunciaban que aunque se estaba produciendo un cambio a nivel de cobertura, existía una mayor atención por la mujer deportista, sin embargo este cambio era superficial.

The mass media have been used as one means of resisting ideological change, as media practices, production, content and messages continue to perpetuate notions of sexual difference, gender difference, and gender hierarchy. The media have transformed the meanings of women's physicality - women becoming active agents with and of their own bodies and women using their bodies in skilled, physical activity-to commodification, sexuality and femininity.

Coakley (2004), en 1998, destacaba que el hecho de que los medios de comunicación, y en concreto la prensa deportiva, ignore ciertos aspectos de la participación femenina en el deporte influye en la opinión del público a la hora de valorar a las deportistas femeninas. Cunningham, Sagas, Satore, Amsden, y Schellhase (2004: 861) ańadieron que si las niñas y las mujeres no están representadas de manera equitativa por los medios de comunicación, entonces las niñas no gozan de ejemplos necesarios para emular. Así, como resultado de ello, existe la posibilidad de un descenso en la futura participación femenina en el deporte (Pedersen, 2002).

Fink y Kensicki (2002) realizan un estudio donde analizan la construcción de género en publicaciones estadounidenses cuya audiencia principal es la mujer, Sports Illustrated y Sports Illustrated for Women. Según estos autores los artículos diferenciaban a la atleta femenina del masculino con historias donde se destacaban los estereotipos femeninos, y donde el atractivo físico primaba sobre la superioridad atlética. En él destacan que los medios retratan a las mujeres deportistas como objetos sexuales o les atribuyen roles que no son propios del deporte (madre o esposa).

Duncan y Messner, en ocasiones junto a otros investigadores, $(1990,1991,1994,2000)$ han centrado su labor investigadora en el género y la sociología del deporte, prestando especial atención a la cobertura televisiva en EE.UU. Sus trabajos son obras de referencia a la hora de analizar la presencia de la mujer en la información deportiva y la imagen que los medios transmiten de ella.

Frideres y Palao (2006), en un estudio sobre los modelos transmitidos a través de periódicos digitales en EE.UU. y Es- paña destacan que por cada noticia referida a las mujeres hay quince noticias referidas a los hombres.

Angulo (2007) en su estudio Las imágenes de las deportistas en los medios de comunicación introduce el análisis de prensa deportiva, en concreto, analiza Marca, As y Mundo Deportivo. Teniendo en cuenta que no analiza las páginas de los diarios dedicadas al fútbol - lo que supone más de un $50 \%$-, y que su estudio se centra en dos semanas concretas de 2007, los datos son relevantes, sólo un $8,61 \%$ de las informaciones las protagonizan mujeres, frente al $91,39 \%$ de las informaciones protagonizadas por el hombre, y respecto a las fotografías un $7 \%$ representaban a mujeres deportistas, periodistas o dirigentes deportivas, de un total de 1.586 fotos de deporte polideportivo (sin fútbol).

Estudio sobre género y deporte en televisión, realizado por el Consejo Audiovisual de Andalucía (CAA) (2008) destaca que solo el 4,75\% de las emisiones televisivas de deporte corresponden a deporte practicado por mujeres, frente al $90,15 \%$ de deporte masculino, perteneciendo el resto, un $5,11 \%$, al deporte mixto.

Al revisar el Proyecto de Monitoreo Global de Medios (GMMP) en el caso concreto de España (WACC, 2000, 2005, 2010), en el ańo 2009 las mujeres representan el 23\% de las personas que aparecen en la información, un punto por debajo del nivel mundial. Sin embargo, cabe destacar que aunque se ha producido un aumento general en todos los sectores informativos desde el año 1999, en el caso de la información deportiva ha descendido. Mientras que en el año 1999 las mujeres solo fueron sujeto de las noticias deportivas en el $9 \%$ de los casos, en el 2004 aumentó hasta el 16\% para caer hasta el $11 \%$ en el 2009.

A este respecto se refiere López-Díez (2011), analiza el estado de la cuestión manejando diferentes estudios sobre el tratamiento de la información, centrándose en los datos que tienen que ver con mujer y deporte. La aportación más valiosa es la realización de una clasificación de los estereotipos de la mujer deportista, para concluir con una serie de recomendaciones a los medios.

Sainz de Baranda (2013) en su estudio longitudinal de la prensa deportiva, Mujeres y deporte en los medios de comunicación, concluye que la imagen de las mujeres que la prensa deportiva española transmite está altamente desequilibrada respecto del hombre. La mujer es sujeto noticioso únicamente en el $5,11 \%$ de los casos - unas veces sola $(2,18 \%)$ y otras acompańada por al menos un protagonista masculino $(2,93 \%)$-, mientras que el hombre lo es en el 92,24\%. Este porcentaje no varía con el paso de los años.

El objetivo principal de este estudio es definir los perfiles de mujer que aparecen en las informaciones de la prensa deportiva y como se presentan en la información. 


\section{Metodo}

\section{Muestra, instrumento y procedimiento}

Para el estudio observacional de las protagonistas de la prensa deportiva, se ha utilizado la metodología observacional propuesta por Anguera (2011).

Para definir los perfiles de mujer que protagonizan la prensa deportiva se analizaron todas las informaciones donde al menos aparecía una mujer $(\mathrm{n}=4877)$ que se publicaron durante una semana de duración desde el año 1979 hasta el año 2010 en los periódicos Marca, As, Mundo Deportivo y Sport.

El instrumento utilizado fue "La guía de análisis de prensa deportiva” propuesta por Sainz de Baranda (2013) con índices de fiabilidad muy altos (Kappa $=0.94$, en el peor de los casos).

Partiendo del protagonista como unidad de análisis, pertenezcan o no a la misma información, en primer lugar se recogió el número total de protagonistas - un máximo de ocho - por información y el género de las personas que aparecen en la información. Además por cada una de las protagonistas se han recogido los siguientes datos: si se trata de una protagonista principal o secundaria; la ocupación o cargo, si la persona está descrita con dos ocupaciones, se elige la ocupación que parece más relevante en el contexto de la información y en el caso de personas que son muy conocidas para el público en general codifica la ocupación aún si no se evidencia del contexto de la información (no mencionado, deportista, equipo técnico; árbitra, miembros de comités, federaciones, celebridades / deportistas jubiladas, directivas de clubes, realeza, profesionales de los medios, periodistas, profesionales de las ciencias de la salud, funcionarias del gobierno, académicas, profesionales de la educación, profesionales de las ciencias o tecnología, otros, neutro); su función en la información (tema, portavoz, experta o comentarista, experiencia personal, testigo, opinión popular u otros); si existen relaciones familiares mencionadas (si está descrita en algún sitio del artículo, en términos de una relación familiar: sí, no); cómo presenta la información a esta persona o a la acción de esta persona en la información (heroína, éxito, fracaso, decepción [antiheroína; ángel caído], víctima, superviviente, ninguna de las anteriores); $y$, por último, si la protagonista está directamente citada en el artículo (sí, no).

El análisis estadístico incluyó estadística descriptiva, frecuencias y porcentajes de la información recopilada. Se realizó un análisis inferencial, se utilizaron diferentes pruebas estadísticas, para analizar las posibles diferencias y relaciones entre las distintas variables objeto de estudio. En concreto se utilizó las pruebas T de Student para muestras independientes, la prueba ANOVA de un factor (posc hoc Shefee) y la prueba Chi cuadrado de Pearson. El nivel de significación se fijó en $\mathrm{p} \leq .05$.

\section{Resultados}

En general, la media de protagonistas de las informaciones de la prensa deportiva en las que aparecen Mujeres es 4,29. Destacan las medias de Sport, más de medio punto inferior, y de Mundo Deportivo, cuatro décimas por encima. Los datos que se aprecian en la tabla 1 reflejan diferencias estadísticamente significativas en el número de protagonistas de las informaciones donde al menos aparece una mujer por medio $\left(\mathrm{F}_{3,4876}=\right.$ 31.09, $\mathrm{p}=.001$ ). En concreto, se apreciaron diferencias entre Sport y Mundo Deportivo con el resto de los medios ( $\mathrm{p}=.001)$.

Tabla 1. Media de protagonistas de las informaciones de Mujer por medio.

\begin{tabular}{ll}
\hline & MEDIA \\
\hline Marca & $4,09 \pm 2,52$ \\
As & $4,29 \pm 2,44$ \\
Sport & $3,64 \pm 2,45$ \\
Mundo Deportivo & $4,69 \pm 2,58$ \\
\hline Total & $4,29 \pm 2,53$ \\
\hline
\end{tabular}

Sorprende, tratándose solo de las informaciones donde aparecen mujeres, que del total de los protagonistas analizados ( $\mathrm{n}=$ 20928 ) solo el $58,27 \%$ son mujeres $(n=12195)$.

Cuando se analiza la ocupación o cargo de las mujeres protagonistas de la información deportiva (tabla 2), se observa que las protagonistas femeninas son fundamentalmente deportistas $(68,07 \%)$ y equipos femeninos $(22,62 \%)$, es lo propio en el ámbito en el que aparecen.

Sin embargo, llama la atención que la frecuencia de la ocupación de las protagonistas que les siguen no pertenezca al 'Ámbito Deportivo' y que en un 2,7\% no se mencione dentro de la información.

Al analizar la evolución de las diferentes ocupaciones de las protagonistas, se observa que la evolución de las deportistas es inversamente proporcional a la evolución de los equipos femeninos como protagonistas de las informaciones.

Además, la frecuencia de las mujeres que protagonizan las informaciones y su ocupación no se menciona marca una tendencia al descenso, fundamentalmente en los ańos centrales. Algo similar ocurre con las famosas que suelen ocupar las páginas del papel cuché.

Las protagonistas pertenecientes a la realeza se comportan de manera inversa, marcando sus índices más altos en los ańos noventa, por encima del $1 \%$.

Las protagonistas miembros de comités o federaciones y las funcionarias del gobierno o políticas que aparecen en el primer período por encima del $0,5 \%$ descienden durante los ańos siguientes. Pero a principios del siglo XXI se produce un aumento de su presencia de más de un punto, y en el período 2005-2009 la frecuencia vuelve a disminuir: el porcentaje de 
funcionarias del gobierno o políticas queda en un $1 \%$ y el de miembros de comités o federaciones en un $0,33 \%$.

En el resto de las ocupaciones, siempre en porcentajes muy bajos, se detecta un incremento de su presencia a partir del siglo XXI, estas son: profesionales de los medios y de las ciencias de la salud, académicas, celebridades y deportistas retiradas, directivas de clubes, arbitras y otras ocupaciones.

Tabla 2. Evolución de la ocupación de las protagonistas femeninas.

\begin{tabular}{|c|c|c|c|c|c|c|c|c|c|}
\hline & 1979 & $\begin{array}{l}1980- \\
1984 \\
\end{array}$ & $\begin{array}{l}1985- \\
1989\end{array}$ & $\begin{array}{l}1990- \\
1994 \\
\end{array}$ & $\begin{array}{l}1995- \\
1999\end{array}$ & $\begin{array}{l}2000- \\
2004 \\
\end{array}$ & $\begin{array}{l}2005- \\
2009\end{array}$ & 2010 & TOTAL \\
\hline Deportista & 58,43 & 64 & 71,28 & 74,15 & 73,22 & 64,85 & 57,7 & 63,38 & 68,07 \\
\hline Equipo & 33,71 & 26,5 & 20,83 & 19,28 & 19,08 & 23,31 & 27,31 & 24,78 & 22,62 \\
\hline No mencionado & 3 & 2,72 & 2,71 & 2,34 & 2,13 & 3,35 & 2,95 & 4,17 & 2,7 \\
\hline Famosas/celebridades & 3,75 & 3,7 & 2,39 & 0,59 & 1,32 & 1,94 & 3,61 & 3,95 & 2,25 \\
\hline Otros & 0,37 & 0,51 & 0,28 & 0,42 & 0,9 & 1,12 & 2,07 & 0,44 & 0,77 \\
\hline Realeza & 0 & 0,21 & 0,83 & 1,38 & 1,18 & 0,52 & 0,27 & 0 & 0,75 \\
\hline $\begin{array}{l}\text { Celebridades / } \\
\text { Deportistas retiradas }\end{array}$ & 0 & 0,51 & 0,18 & 0,38 & 0,61 & 0,52 & 2,01 & 0 & 0,6 \\
\hline $\begin{array}{l}\text { Funcionarias del Gobierno, } \\
\text { Políticas }\end{array}$ & 0 & 0,72 & 0,14 & 0,13 & 0,33 & 1,71 & 1 & 0,88 & 0,57 \\
\hline Equipo técnico & 0 & 0,26 & 0,64 & 0,67 & 0,28 & 0,52 & 0,87 & 0,44 & 0,52 \\
\hline $\begin{array}{l}\text { Miembros de Comités, } \\
\text { Federaciones }\end{array}$ & 0,37 & 0,51 & 0,37 & 0,33 & 0,19 & 1,27 & 0,33 & 0,22 & 0,44 \\
\hline Directivas de clubes & 0 & 0,1 & 0,09 & 0,13 & 0,28 & 0,3 & 0,47 & 0,44 & 0,21 \\
\hline Profesionales de los Medios & 0,37 & 0,1 & 0,05 & 0,13 & 0,09 & 0,37 & 0,4 & 0,22 & 0,17 \\
\hline Árbitra & 0 & 0 & 0 & 0,04 & 0,33 & 0,07 & 0,67 & 0,22 & 0,16 \\
\hline Profesionales de las Ciencias de la Salud & 0 & 0,05 & 0,18 & 0 & 0 & 0 & 0,07 & 0,66 & 0,07 \\
\hline Policía, militares, seguridad & 0 & 0,1 & 0 & 0,04 & 0,05 & 0,07 & 0,07 & 0,22 & 0,06 \\
\hline Académicas & 0 & 0 & 0,05 & 0 & 0 & 0,07 & 0,2 & 0 & 0,04 \\
\hline
\end{tabular}

Tras el análisis de los datos de la tabla 2 se han definido dos perfiles de mujer que protagonizan las informaciones de la prensa deportiva. Se ha procedido a agrupar a las protagonistas femeninas en las que pertenecen al 'Ámbito Deportivo' y las que no pertenecen a este ámbito, las 'Invitadas'. La codificación queda de la siguiente manera:

- 'Ámbito Deportivo': deportista, equipo, equipo técnico, miembros de comités y federaciones, directivas de clubes, árbitra y celebridades o deportistas retiradas.

- 'Invitadas': famosas y celebridades, realeza, funcionarias del gobierno y políticas, profesionales de los medios, profesionales de las ciencias de la salud, policía, militares y seguridad, académicas, otros y no mencionado.
Según esta clasificación los porcentajes de las protagonistas que pertenecen al 'Ámbito Deportivo' (92,63\%) son muy superiores a las de 'Invitadas' al ámbito (7,37\%). Sin embargo, al analizar la evolución de las protagonistas pertenecientes al 'Ámbito Deportivo' y las 'Invitadas' durante los 32 años analizados, se observa que las 'Invitadas' ganan protagonismo fundamentalmente en el siglo XXI (tabla 3).

Claramente se produce una tendencia al descenso en los últimos quince años del análisis de las protagonistas del 'Ámbito Deportivo', y por oposición el aumento de las 'Invitadas'.

El porcentaje de las protagonistas de 'Ámbito Deportivo' aumenta en las décadas de los ochenta y los noventa, sin embargo, a principios del siglo XXI disminuye y en los últimos seis años del análisis su frecuencia se sitúa en torno al 89\%, su cifra más baja.

Tabla 3.- Evolución de las protagonistas según su pertenencia, o no, al ámbito deportivo

\begin{tabular}{|c|c|c|c|c|c|c|c|c|c|}
\hline & 1979 & $1980-1984$ & $1985-1989$ & 1990-1994 & 1995-1999 & $2000-2004$ & $2005-2009$ & 2010 & TOTAL \\
\hline 'Ámbito Deportivo' & 92,51 & 91,88 & 93,39 & 94,98 & 94 & 90,84 & 89,36 & 89,47 & 92,63 \\
\hline 'Invitadas' & 7,49 & 8,12 & 6,61 & 5,02 & 6 & 9,16 & 10,64 & 10,53 & 7,37 \\
\hline
\end{tabular}


Los datos de la tabla 3 indican relaciones estadísticamente significativas entre las variables pertenencia al ámbito deportivo y la evolución temporal $(\chi 2(7, \mathrm{~N}=12195)=65,014$, $\mathrm{p}=.001)$.

De todas las protagonistas se ha analizado el tipo de protagonismo que desarrollan en la información: en un 69,9\% las mujeres son protagonistas principales y un $30,1 \%$ son secundarias. Los datos de la tabla 4 analizan el tipo de protagonismo según la pertenencia, o no, al ámbito deportivo.
Un $71,46 \%$ de las protagonistas femeninas que pertenecen al 'Ámbito Deportivo' son protagonistas principales y el resto son secundarias. En el caso de las protagonistas 'Invitadas' el porcentaje está más igualado, hay más principales que secundarias, pero por solo unas décimas.

Si se analiza la tabla según el tipo de protagonismo, las protagonistas pertenecientes al 'Ámbito Deportivo' se sitúan por encima de la media como protagonistas principales.

Tabla 4. Tipo de protagonismo de las mujeres según su pertenencia, o no, al ámbito deportivo.

\begin{tabular}{llllll}
\hline & \multicolumn{2}{c}{ 'Ámbito Deportivo' } & \multicolumn{2}{c}{ 'Invitadas' } & \multicolumn{1}{c}{ TOTAL } \\
\hline & $\%$ del n columna & $\%$ fila & \% del n columna & $\%$ fila & \% del n columna \\
\hline Principal & 71,46 & 94,7 & 50,28 & 5,3 & 69,9 \\
Secundario & 28,54 & 87,82 & 49,72 & 12,18 & 30,1 \\
\hline Total & 100 & 92,63 & 100 & 7,37 & 100 \\
\hline
\end{tabular}

Los datos de la tabla 4 indican relaciones estadísticamente significativas entre las variables pertenencia al ámbito deportivo y tipo de protagonismo $(\chi 2(1, \mathrm{~N}=12195)=177,552$, $\mathrm{p}=.001)$.

La tabla 5 muestra la evolución de las protagonistas principales y secundarias según su pertenencia, o no, al ámbito deportivo.

Respecto a la evolución de las protagonistas del 'Ámbi- to Deportivo' las principales dominan en cualquier período sobre las secundarias. En el caso de las 'Invitadas' las principales evolucionan de manera ascendente, fundamentalmente en el siglo XXI.

En ambos casos las protagonistas principales marcan una tendencia clara al ascenso que en el caso de las 'Invitadas' es más pronunciada, aunque no llega a ocasionar la misma proporción.

Tabla 5. Evolución del tipo de protagonismo de las mujeres según su pertenencia, o no, al ámbito deportivo.

\begin{tabular}{llllllllll}
\hline & & 1979 & $1980-1984$ & $1985-1989$ & $1990-1994$ & $1995-1999$ & $2000-2004$ & $2005-2009$ & 2010 \\
\multirow{2}{*}{ 'Ámbito Deportivo' } & Principal & 75,3 & 67,97 & 70,09 & 73,84 & 70,95 & 66,89 & 76,48 & 77,7 \\
& Secundaria & 24,7 & 32,03 & 29,91 & 26,16 & 29,05 & 33,11 & 23,52 & 22,3 \\
\hline \multirow{2}{*}{ 'Invitadas' } & Principal & 30 & 41,77 & 34,72 & 47,5 & 54,33 & 63,41 & 63,52 & 52,08 \\
& Secundaria & 70 & 58,23 & 65,28 & 52,5 & 45,67 & 36,59 & 36,48 & 47,92 \\
\hline
\end{tabular}

En general la función principal de las mujeres en las informaciones es ser el tema de la misma, como se observa en el total de la tabla 6. Otras funciones reflejadas son las de portavoz, con solo un $0,21 \%$, y la de experiencia personal que supone un $0,02 \%$ de los casos. En ningún caso se ha analizado una información cuyo protagonista sea testigo, experta o comentarista o que refleje la opinión popular de una mujer. Sin embargo, en el 0,86\% no se sabe su función en la información.

Si analizamos ambos perfiles, las pertenecientes al 'Ámbito Deportivo' son el tema de la información en el 99,66\% de los casos y las 'Invitadas' lo son en el $89,43 \%$. Por lo tanto las 'Invitadas' ejercen de portavoz en un 1,22\% de los casos, con mayor frecuencia que las de 'Ámbito Deportivo'. Pero también se registra un alto porcentaje de protagonistas 'Invitadas' de las que no se sabe cuál es su función, el por qué están en la información, esto ocurre en un 9,01\% de los casos.

$\mathrm{Al}$ analizar la distribución de las funciones en la información según la pertenecía o no al 'Ámbito Deportivo' de las protagonistas, destaca que las de 'Ámbito Deportivo' superan la media en experiencia personal $(100 \%$, aunque se trate de la función menos frecuente) y tema (93,33\%). Por el contrario, las 'Invitadas' se sitúan por encima de la media en portavoz $(42,31 \%)$ y cuando no se sabe cuál es su función en la noticia $(80 \%)$. 
Tabla 6. Función en la información de las mujeres según su pertenencia, o no, al ámbito deportivo.

\begin{tabular}{llllll}
\hline & \multicolumn{2}{c}{ 'Ámbito Deportivo' } & \multicolumn{2}{c}{ 'Invitadas' } & Total \\
\hline FUNCIONES & \% del N columna & $\%$ fila & \% del N columna & $\%$ fila & \% del N columna \\
\hline Tema & 99,66 & 93,33 & 89,43 & 6,67 & 98,91 \\
No sabe & 0,19 & 20 & 9,34 & 80 & 0,86 \\
$\begin{array}{l}\text { Portavoz } \\
\begin{array}{l}\text { Experiencia per- } \\
\text { sonal }\end{array}\end{array}$ & 0,13 & 57,69 & 1,22 & 42,31 & 0,21 \\
\hline Total & 0,02 & 100 & 0 & 0 & 0,02 \\
\hline
\end{tabular}

Si analizamos la evolución de la función en la información de las protagonistas según su pertenencia, o no, al ámbito deportivo (tabla 7), queda claro que la función de las protagonistas de 'Ámbito Deportivo' claramente es ser el tema de la misma, su frecuencia mínima es del 99,33\% en el período comprendido entre 1980 y 1984.

En el caso de las protagonistas 'Invitadas' se observa una tendencia ascendente y constante en el tiempo de la función tema, en detrimento de que no se sabe cuál es su función dentro de la información.

Tabla 7. Evolución de la función en la información de las protagonistas según su pertenencia, o no, al ámbito deportivo.

\begin{tabular}{|c|c|c|c|c|c|c|c|c|c|}
\hline \multirow{5}{*}{ 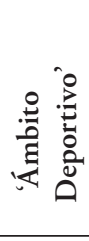 } & & 1979 & $1980-1984$ & $1985-1989$ & $1990-1994$ & 1995-1999 & $2000-2004$ & 2005-2009 & 2010 \\
\hline & Tema & 99,6 & 99,33 & 100 & 99,6 & 99,7 & 99,43 & 99,78 & 100 \\
\hline & No sabe & 0,4 & 0,56 & 0 & 0,09 & 0,15 & 0,33 & 0,07 & 0 \\
\hline & Portavoz & 0 & 0,11 & 0 & 0,31 & 0,05 & 0,25 & 0,15 & 0 \\
\hline & Experiencia personal & 0 & 0 & 0 & 0 & 0,1 & 0 & 0 & 0 \\
\hline \multirow{4}{*}{ 苞 } & Tema & 70 & 77,85 & 80,56 & 96,67 & 96,06 & 95,12 & 94,34 & 95,83 \\
\hline & No sabe & 30 & 20,89 & 18,06 & 3,33 & 3,15 & 1,63 & 4,4 & 4,17 \\
\hline & Portavoz & 0 & 1,27 & 1,39 & 0 & 0,79 & 3,25 & 1,26 & 0 \\
\hline & Experiencia personal & 0 & 0 & 0 & 0 & 0 & 0 & 0 & 0 \\
\hline
\end{tabular}

En la tabla 8 se observa que un $5,16 \%$ se mencionan relaciones familiares de las mujeres en el texto de las informaciones. Si se diferencian los datos en función a los dos perfiles de mujeres que aparecen en la prensa deportiva las relaciones familiares se mencionan en un $2,12 \%$ cuando la protagonista pertenece al 'Ámbito Deportivo' y un 43,27\% en el caso de las 'Invitadas'.

La frecuencia de la mención de las relaciones familiares de las protagonistas es claramente superior en las 'Invitadas' (61\%) que en las de 'Ámbito Deportivo' (38,16\%).

Tabla 8. Relaciones familiares mencionadas de las protagonistas según su pertenencia, o no, al ámbito deportivo.

\begin{tabular}{llllll}
\hline & \multicolumn{2}{l}{ 'Ámbito Deportivo' } & \multicolumn{2}{l}{ 'Invitadas' } & \multicolumn{2}{l}{ Total } \\
\hline RELACIONES FAMILIARES & \% del N columna & \% fila & \% del N columna & \% fila & \% del N columna \\
\hline Sí & 2,12 & 38,16 & 43,27 & 61,84 & 5,16 \\
No & 97,88 & 95,59 & 56,73 & 4,41 & 94,84 \\
\hline Total & 100 & 92,63 & 100 & 7,37 & 100 \\
\hline
\end{tabular}

Los datos de la tabla 8 indican relaciones estadísticamente significativas entre las variables pertenencia al ámbito deportivo y mención de relaciones familiares $(\chi 2(1, \mathrm{~N}=12195)=$ $2881,909, \mathrm{p}=.001)$.
Queda claro que gran parte de las 'Invitadas' lo son por mantener relaciones familiares con otros/as protagonistas (figura 1). 
Figura 1. Ejemplo de mención de relaciones familiares en la información (diario Sport, 18-02-1999)
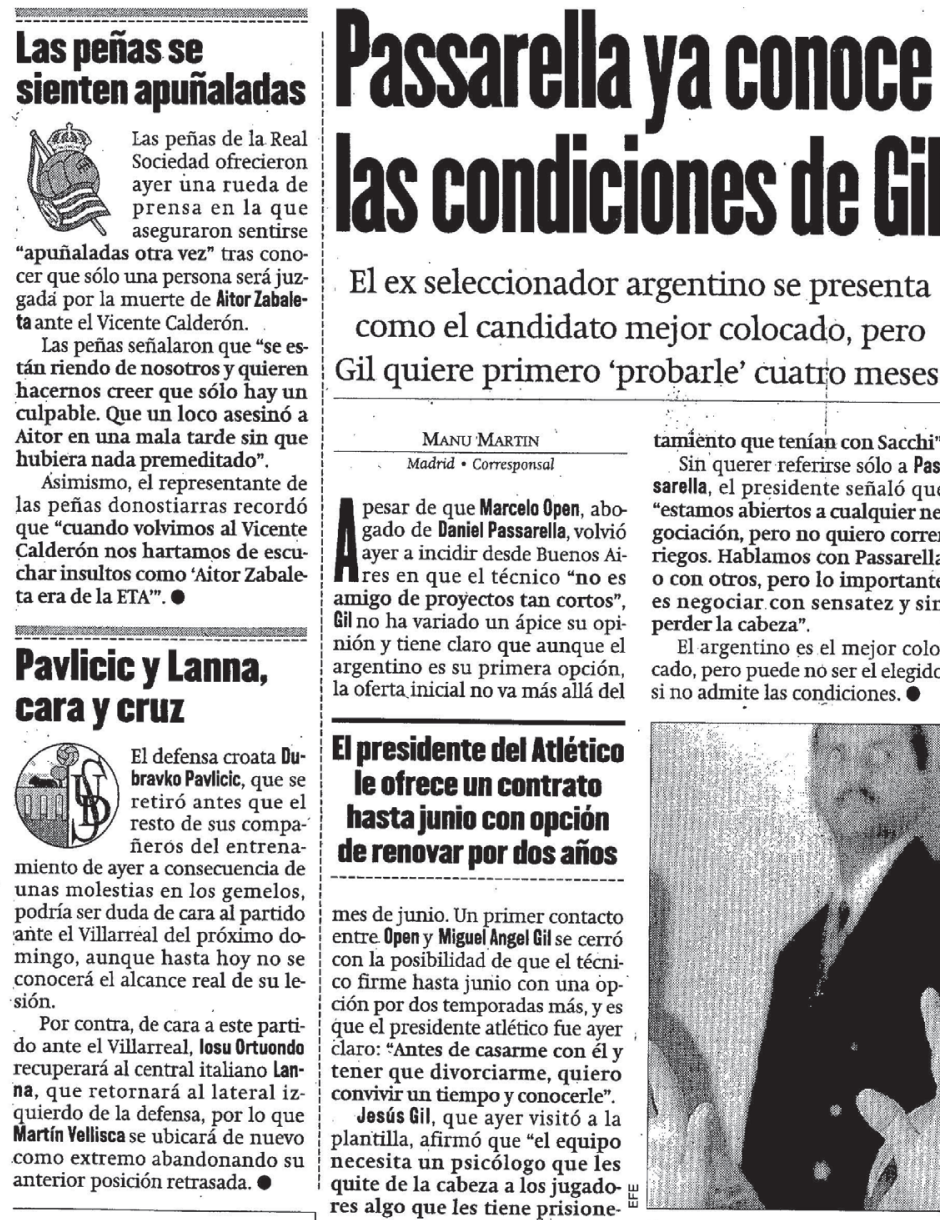

El ex seleccionador argentino se presenta como el candidato mejor colocado, pero Gil quiere primero 'probarle' cuatro meses.

\begin{tabular}{|c|c|}
\hline MANU MARTIN & tamiento que tenían con Sacchi" \\
\hline $\begin{array}{l}\text { Madrid - Corresponsal } \\
\text { pesar de que Marcelo Open, abo- } \\
\text { gado de Daniel Passarella, volvió } \\
\text { ayer a incidir desde Buenos Ai- } \\
\text { res en que el técnico "no es } \\
\text { amigo de proyectos tan cortos", } \\
\text { Gil no ha variado un ápice su opi- } \\
\text { nión y tiene claro que aunque el } \\
\text { argentino es su primera opción, } \\
\text { la oferta inicial no va más allá del }\end{array}$ & $\begin{array}{l}\text { Sin querer referirse sólo a Pas- } \\
\text { sarella, el presidente señaló que } \\
\text { "estamos abiertos a cualquier ne } \\
\text { gociación, pero no quiero correr } \\
\text { riegos. Hablamos con Passarella } \\
\text { o con otros, pero lo importante } \\
\text { es negociar con sensatez y sin } \\
\text { perder la cabeza". } \\
\text { El argentino es el mejor colo- } \\
\text { cado, pero puede no ser el elegido } \\
\text { si no admite las condiciones. }\end{array}$ \\
\hline
\end{tabular}

\section{LAS FRASES}

Necesidades

"El equipo precisa

de un psicólogo

que le quite el

agarrotamiento que

tenía con Sacchi"

\section{Tranquilidad}

"Lo importante

es negociar con

sensatez y sin

perder la cabeza"

\section{Plazo}

"Antes de casarme

con un técnico,

quiero convivir un

tiempo y conocerle"

\section{El presidente del Atlético} le ofrece un contrato hasta junio con opción de renovar por dos años

mes de junio. Un primer contacto entre Open y Miguel Angel Gil se cerro con la posibilidad de que el técnico firme hasta junio con una opción por dos temporadas más, y es que el presidente atlético fue ayer claro: "Antes de casarme con él y tener que divorciarme, quiero convivir un tiempo y conocerle". Jesús Gil, que ayer visitó a la plantilla, afirmó que "el equipo necesita un psicólogo que les quite de la cabeza a los jugadores algo que les tiene prisione

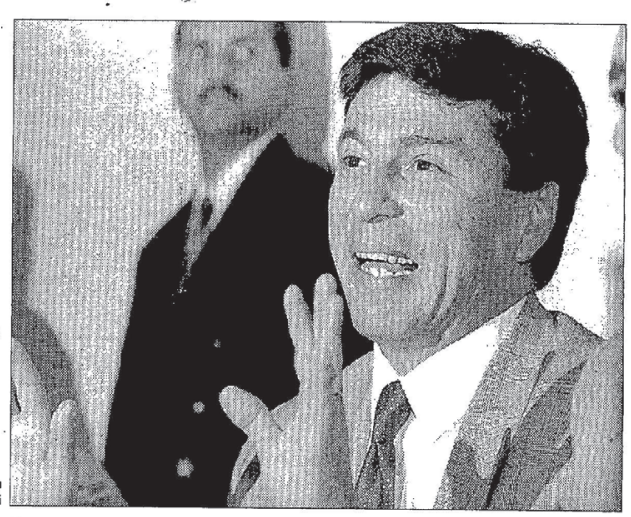
ros. Hay que quitarles el agarro-

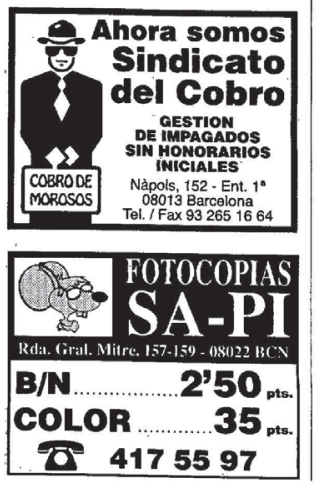
mujeres de los jugadores

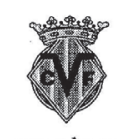

El fisiotera- óptimas".

peuta del opt Para Prumboom, ju- saturación d

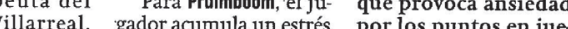
Loo Prumbo- más fuerte de lo habi- por los putos en jueom, malmb fuerte de lo habi- go, ya que es ahora ayer a las mujeres de los "versas cuusas, pero prin- cidir la completición", jugadores parar tos cisas causas, pero prin- cidir la competición jugadoma de los futbo cipalmenté por dos: la explico Pruimboum. listas en febros duro acum y les pidió que sean ca- ción y la nutrición. y les pidió que sean ca- ción y la nutricion. res de los jugadores es nes psicoemocionales : a Liga se llega con una crear buen ambiente. El 'fisio' se reúne con las
En la tabla 9 se observa la evolución de las relaciones familiares mencionadas en el texto de la información según la pertenencia, o no, al ámbito deportivo de las protagonistas. En general la mención de relaciones familiares en las informaciones marca una tendencia ascendente en ambos casos, muy superior en el caso de las 'Invitadas' que aumentan más de veinte puntos. La frecuencia más alta en el caso de las 'Invitadas' se produce en el período 2000-2004 (53,66\%).

Las protagonistas pertenecientes al 'Ámbito Deportivo', aunque aumentan las menciones de las relaciones familiares a lo largo del tiempo, se mantienen en frecuencias muy bajas, marcando la más alta en el período 2005-2009 (3,22\%). 
Tabla 9. Evolución de las relaciones familiares mencionadas de las protagonistas según su pertenencia, o no, al ámbito deportivo.

\begin{tabular}{lllllllll}
\hline $\begin{array}{l}\text { Relaciones familiares } \\
\text { mencionadas }\end{array}$ & 1979 & $1980-1984$ & $1985-1989$ & $1990-1994$ & $1995-1999$ & $2000-2004$ & $2005-2009$ & 2010 \\
& 1,21 & 1,57 & 2,11 & 2,2 & 1,56 & 2,95 & 3,22 & 1,47 \\
& 98,79 & 98,43 & 97,89 & 97,8 & 98,44 & 97,05 & 96,78 & 98,53 \\
\hline & 25 & 34,18 & 41,67 & 40,83 & 48,82 & 53,66 & 44,65 & 45,83 \\
\hline & 75 & 65,82 & 58,33 & 59,17 & 51,18 & 46,34 & 55,35 & 54,17 \\
\hline
\end{tabular}

Teniendo en cuenta que la información y modelos de referencia que los jóvenes obtienen sobre la práctica deportiva la consiguen a través de los distintos medios de comunicación, es importante resaltar como la prensa deportiva presenta a las mujeres, o a sus acciones, en las informaciones (tabla 10).

En general, la presentación de las mujeres, o sus acciones, se presentan vinculas al éxito en un 66,35\% y vinculadas al fracaso en un $11,5 \%$. La frecuencia de su presentación como heroínas, o en acciones heroicas, es muy baja (0,53\%), pero también lo son las vinculadas a la decepción, anti-heroína $(0,25 \%)$. Su vinculación como víctima se ha producido en un $2,44 \%$ de la muestra, y como una superviviente en el $0,01 \%$.
En el $18,93 \%$ de los casos no se presenta en ninguna de las demás situaciones.

Al diferenciar entre los dos perfiles de mujer definidos se observa que las mujeres pertenecientes al 'Ámbito Deportivo' superan a las de 'Invitadas' en su presentación, o de sus acciones, en todos los casos, como es lógico puesto que la muestra total no es equilibrada. Sin embargo, las 'Invitadas' se sitúan por encima de la media en los casos de presentación como víctima $(23,91 \%)$ y como heroínas $(12,31 \%)$. también superan la media cuando no se identifican con ninguno de los ítems planteados $(22,17 \%)$.

Tabla 10. Presentación de las protagonistas, o sus acciones, en la información según su pertenencia, o no, al ámbito deportivo

\begin{tabular}{llllll}
\hline & 'Ámbito Deportivo' & 'Invitadas' & & Total \\
\hline PRESENTACIÓN & $\%$ del N columna & $\%$ fila & \% del N columna & $\%$ fila & \% del N columna \\
\hline Éxito & 69,08 & 96,44 & 32,04 & 3,56 & 66,35 \\
Fracaso & 12,25 & 98,72 & 2 & 1,28 & 11,5 \\
\hline Heroína & 0,5 & 87,69 & 0,89 & 12,31 & 0,53 \\
\hline Decepción & 0,25 & 93,33 & 0,22 & 6,67 & 0,25 \\
\hline Superviviente & 0,01 & 100 & 0 & 0 & 0,01 \\
\hline Víctima & 2 & 76,09 & 7,9 & 23,91 & 2,44 \\
\hline Ninguna de las demás & 15,91 & 77,83 & 56,95 & 22,17 & 18,93 \\
\hline Total & 100 & 92,63 & 100 & 7,37 & 100 \\
\hline
\end{tabular}

En la tabla 11 los datos muestran la evolución de la presentación de las protagonistas, o sus acciones, en la información según su pertenencia, o no, al ámbito deportivo.

La presentación de las protagonistas pertenecientes al 'Ámbito Deportivo' evoluciona de la siguiente manera: la frecuencia de la actitud de éxito describe una línea descendente, al contrario de la de la actitud de fracaso, aunque su comportamiento es muy heterogéneo. Como heroínas su frecuencia asciende a lo largo del tiempo, por primera vez en la primera década del siglo XXI por encima del 1\% (en el año 2010 vuelve a estar por debajo); la frecuencia de la actitud de decepción no aparece de manera constante y cuando lo hace su frecuencia es muy baja, por debajo del 1\%; aumenta la presentación como víctima (en el año 2010 se sitúa en un 7,35\%) y la de superviviente es anecdótica, solo se registran casos durante el período 1980- 1984 (0,06\%). El mayor descenso se produce en las acciones donde no se describen ninguna de las anteriores, aunque su frecuencia es heterogénea.

La presentación las protagonistas 'Invitadas' evolucionan de manera diferente: la actitud éxito marca una línea ascendente pero heterogénea, la de fracaso también es ascendente, pero es inconstante en el tiempo. Llama la atención que como heroínas solo se recogen casos en el primer (5\%) y último año analizado (14,58\%), pero son frecuencias altas. La actitud de decepción también es anecdótica, solo se registran casos durante el período comprendido entre 2000 y 2004 (1,63\%). 
Asciende la presentación como víctima, aunque su frecuencia es heterogénea. Llama la atención que la presentación en ninguno de los demás casos descritos desciende a lo largo del tiempo. Entre el primer período y el último analizado desciende casi cuarenta puntos, lo que indica que su presencia es más relevante.

Tabla 11. Evolución de la presentación de las protagonistas, o sus acciones, en la información según su pertenencia, o no, al ámbito deportivo

\begin{tabular}{|c|c|c|c|c|c|c|c|c|c|}
\hline & & 1979 & $1980-1984$ & 1985-1989 & 1990-1994 & 1995-1999 & $2000-2004$ & $2005-2009$ & 2010 \\
\hline \multirow{7}{*}{ 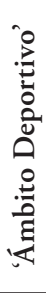 } & Heroína & 0,81 & 0,45 & 0,1 & 0,26 & 0,4 & 1,15 & 1,2 & 0,25 \\
\hline & Éxito & 58,7 & 68,64 & 73,58 & 74,5 & 65,43 & 63,69 & 66,67 & 66,42 \\
\hline & Fracaso & 14,98 & 12,13 & 9,53 & 8,5 & 13,17 & 15,82 & 16,63 & 16,18 \\
\hline & Decepción & 0,81 & 0,17 & 0 & 0,53 & 0,4 & 0 & 0,15 & 0,25 \\
\hline & Víctima & 2,43 & 0,89 & 0,88 & 1,32 & 2,41 & 2,79 & 3,3 & 7,35 \\
\hline & Superviviente & 0 & 0,06 & 0 & 0 & 0 & 0 & 0 & 0 \\
\hline & Ninguna de las demás & 22,27 & 17,66 & 15,91 & 14,88 & 18,19 & 16,56 & 12,06 & 9,56 \\
\hline \multirow{7}{*}{ 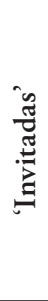 } & Heroína & 5 & 0 & 0 & 0 & 0 & 0 & 0 & 14,58 \\
\hline & Éxito & 20 & 19,62 & 25,69 & 43,33 & 36,22 & 21,95 & 50,31 & 22,92 \\
\hline & Fracaso & 0 & 0 & 0,69 & 2,5 & 0 & 5,69 & 3,77 & 2,08 \\
\hline & Decepción & 0 & 0 & 0 & 0 & 0 & 1,63 & 0 & 0 \\
\hline & Víctima & 5 & 3,16 & 12,5 & 1,67 & 14,96 & 6,5 & 7,55 & 12,5 \\
\hline & Superviviente & 0 & 0 & 0 & 0 & 0 & 0 & 0 & 0 \\
\hline & Ninguna de las demás & 70 & 77,22 & 61,11 & 52,5 & 48,82 & 64,23 & 38,36 & 47,92 \\
\hline
\end{tabular}

Los datos de la tabla 12 muestran que, en general, solo en el $5.69 \%$ las protagonistas están directamente citadas en el artículo.

En el caso de las mujeres que pertenecen al 'Ámbito Deportivo’ están directamente citadas en la información en un $5,55 \%$ y las 'Invitadas' lo están en un 10,79\%.
En general, al analizar todas las mujeres que están citadas el porcentaje de las que pertenecen al 'Ámbito Deportivo' $(86,6 \%)$ se sitúa por debajo de la media.

Tabla 12. Citas de las protagonistas en la información según su pertenencia, o no, al ámbito deportivo.

\begin{tabular}{llllll}
\hline \multicolumn{3}{l}{ 'Ámbito Deportivo' } & 'Invitadas' & & Total \\
\hline CITAS & $\%$ del N columna & $\%$ fila & \% del N columna & \% fila & \% del N columna \\
\hline Sí & 5,55 & 86,6 & 10,79 & 13,4 & 5,94 \\
No & 94,45 & 93,01 & 89,21 & 6,99 & 94,06 \\
\hline Total & 100 & 92,63 & 100 & 7,37 & 100 \\
\hline
\end{tabular}

Los datos de la tabla 12 indican relaciones estadísticamente significativas entre las variables pertenencia al ámbito deportivo y las citas $(\chi 2(1, \mathrm{n}=12195)=40,930, \mathrm{p}=.001)$.

En la tabla 13 los datos muestran un comportamiento muy diferente en la evolución de las protagonistas citadas en la información según su pertenencia, o no, al ámbito deportivo.

Las protagonistas que pertenecen al 'Ámbito Deportivo' siguen una evolución ascendente y constante durante todos los años en el porcentaje de citas en el texto periodístico. Al comparar el año $1979(1,62 \%)$ y el 2010 (6,37\%) se confirma la tendencia ascendente.
Las protagonistas 'Invitadas' tienen un comportamiento heterogéneo a lo largo del tiempo, con fuertes subidas y bajadas, pero en general la línea de tendencia es descendente. A principios de los ochenta comienzan con un $17,72 \%$ de citas y desciende hasta principios de los noventa hasta un 3,33\%. Durante los ańos siguientes asciende y comienza el siglo XXI con un 19,51\%, su índice más alto, vuelve a descender en el período siguiente $(6,92 \%)$.

Lo que está claro es que las frecuencias de las 'Invitadas' citadas en los textos informativos es superior a la frecuencia de las de 'Ámbito Deportivo' durante todos los ańos del análisis excepto el período 1990-1994. 
Tabla 13. Evolución de las citas de las protagonistas en la información según su pertenencia, o no, al ámbito deportivo.

\begin{tabular}{llllllllll}
\hline & Citas & $\mathbf{1 9 7 9}$ & $\mathbf{1 9 8 0 - 1 9 8 4}$ & $\mathbf{1 9 8 5 - 1 9 8 9}$ & $\mathbf{1 9 9 0 - 1 9 9 4}$ & $\mathbf{1 9 9 5 - 1 9 9 9}$ & $\mathbf{2 0 0 0 - 2 0 0 4}$ & $\mathbf{2 0 0 5 - 2 0 0 9}$ & $\mathbf{2 0 1 0}$ \\
\multirow{2}{*}{ 'Ámbito Deportivo' } & Sí & 1,62 & 2,4 & 4,62 & 5,02 & 7,09 & 7,79 & 8,24 & 6,37 \\
& No & 98,38 & 97,6 & 95,38 & 94,98 & 92,91 & 92,21 & 91,76 & 93,63 \\
\hline \multirow{2}{*}{ 'Invitadas' } & Sí & 15 & 17,72 & 8,33 & 3,33 & 7,87 & 19,51 & 6,92 & 10,42 \\
& No & 85 & 82,28 & 91,67 & 96,67 & 92,13 & 80,49 & 93,08 & 89,58 \\
\hline
\end{tabular}

\section{Discusión}

Que una mujer consiga ser laureada por una conquista deportiva no le garantiza visibilidad en un medio de comunicación por ese logro. En ocasiones captará mayor interés por otras actividades ajenas al deporte.

Que la presencia de los hombres va más allá de las informaciones que protagonizan, se refleja en el número de personajes masculinos de las informaciones donde aparecen mujeres. Sorprende que solo un $58,27 \%$ sean mujeres, en consecuencia destaca el porcentaje tan elevado de hombres, un $40,89 \%$.

Que la mujer aparezca en la información no significa que al lector le sea fácil localizar el texto en el que se habla de ella. Será difícil valorar los logros deportivos porque la jerarquización de la información que determina qué informaciones son más importantes que otras, evidencia que los triunfos femeninos no son relevantes. Un claro ejemplo de esta circunstancia es la siguiente: "Lewis, a una centésima de $\mathrm{Hi}$ nes” (As, 16-05-1983, pág. 24), una crónica sobre el Mundial de atletismo. Dentro de la misma, en dos líneas, se relata el récord femenino de salto de longitud que consigue la atleta Cusmir ${ }^{1}$. Si tenemos en cuenta que es la única deportista dentro de la información que supera un récord a nivel mundial, y que Tom Peatronov, récord Masculino en la misma disciplina tiene un breve para el solo en el mismo diario (pág. 31), será difícil apreciar su logro.

Si a esto se le añade que los Hombres son los entrenadores, no se hace referencia a las Mujeres ni con nombre propio: "Brasa dio la Selección olímpica" (Marca, 7-06-1996 pág. 39), para poder descubrir que se esconde tras este titular, tendremos que profundizar en la lectura y nos revelará que se trata de la Selección española Femenina de Jockey.

Ya se ha comentado que la prensa deportiva no sólo se informa sobre deportistas y mujeres pertenecientes al 'Ámbito Deportivo', también se informa sobre otras mujeres que no pertenecen a dicho ámbito, las 'Invitadas'. Teniendo en cuenta los dos perfiles de mujer que se han tomado como referencia - en función a su pertenencia o no al ámbito deportivo - la relación entre los dos puede ser contemplada cuantitativa

1 La atleta Anisoara Cusmir-Stanciu fue plusmarquista mundial con una marca de 7 metros y 43 centímetros obtenida en 1983 en Bucarest y cualitativamente. Desde el punto de vista cuantitativo, el 92,63\% de las informaciones corresponde a la mujer de 'Ámbito Deportivo' y el 7,37\% al perfil no deportivo, las 'Invitadas'. La tendencia evolutiva es el incremento de este último perfil hasta alcanzar $10,53 \%$. Esta ventaja aparente en favor del perfil deportivo queda matizada desde el punto de vista cualitativo.

En general aumenta el protagonismo principal de las mujeres, pero es más notable en las 'Invitadas'.

La función principal de las mujeres en las informaciones es ser el tema de la misma. Las funciones de testigos, expertas o comentarista o que refleje la opinión popular de una mujer no se han recogido en ningún caso. $\mathrm{Y}$ aunque en algunas ocasiones ejercen como portavoces, son las 'Invitadas' las que lo hacen en mayor proporción.

Esto unido a las pocas voces femeninas citadas en las informaciones deja claro que la mujer sigue sin tener la categoría suficiente para ser personaje y, menos aún, fuente de la noticia.

Las menciones específicas a la situación marital o familiar de las protagonistas en general son bajas. Sin embargo, en el caso de las 'Invitadas' son claramente superiores. Queda claro que gran parte de las 'Invitadas' lo son por mantener relaciones familiares con otros/as protagonistas (43,27\%). Este tipo de menciones aumentan a lo largo del tiempo en ambos casos.

La prensa deportiva presenta a las mujeres, o a sus acciones, en las informaciones vinculadas al éxito en gran parte de los casos. Sin embargo, apenas se recogen casos de acciones heroicas, siendo mayor la proporción en el caso de las 'Invitadas', lo que indica que existen pocos referentes deportivos, modelos de referencia donde los jóvenes puedan verse reflejados.

El aumento de la presencia de las 'Invitadas' a lo largo del tiempo en las páginas de los diarios hace que la invisibilidad de los logros deportivos femeninos aumente.

\section{Conclusiones}

- La presencia del hombre va más allá del número de informaciones que protagoniza: un $41,1 \%$ de los personajes que aparecen en las informaciones de mujer son hombres.

- Existen dos perfiles de mujer en la prensa deportiva: la propia del ámbito deportivo, y ese personaje que hemos dado 
en llamar 'Invitadas', por lo general familiares, parejas, famosas o aficionadas. La mujer acompañante, se podría denominar también. Desde el punto de vista cuantitativo, el $86,8 \%$ de las informaciones corresponde a la mujer de ámbito deportivo y el 13,2\%, al perfil no deportivo.

- Entre los años 1979 y 2010 las 'Invitadas' aumentan su presencia en la prensa deportiva de manera cuantitativa y como personajes principales.

- En general, las mujeres no interesan ni como testigos ni como expertas. En los pocos casos que sí interesan las 'Invitadas' lo son con mayor frecuencia. Queda claro en las frecuencias de las 'Invitadas' citadas en los textos informativos, superior a las de Ámbito Deportivo.
- Teniendo en cuenta que la información y modelos de referencia que los jóvenes obtienen sobre la práctica deportiva la consiguen a través de los distintos medios de comunicación, es importante resaltar la presencia de pocas heroínas, y por lo tanto la prensa deportiva muestra pocos referentes deportivos

- Sin embargo, apenas se recogen casos de acciones heroicas, siendo mayor la proporción en el caso de las 'Invitadas', lo que indica que existen pocos referentes deportivos, modelos de referencia donde los jóvenes puedan verse reflejados.

- En cualquier caso en la prensa deportiva hay pocas heroínas, pocos referentes deportivos.

\section{Referencias}

1. Anguera, Ma. T. (2011). ¿Cómo se lleva a cabo un registro observacional? Revista de Entrenamiento Deportivo, 25 (1), 23-28.

2. Angulo, M. (2007). Las imágenes de las deportistas en los medios de comunicación. Madrid: Ministerio de Educación y Ciencia, Consejo Superior de Deportes.

3. AS. Desde $1979-2010$

4. Asociación para la Investigación de Medios de Comunicación [en línea]. Estudio General de Medios (EGM). http://www.aimc.es/ [consulta: 5 de noviembre de 2012].

5. Coakley, J.J. (2004). Sports in society: Issues and controversies (8th Ed.). New York: McGraw Hill.

6. Consejo Audiovisual de Andalucía (CAA) (2008). Estudio sobre género y deporte en televisión. Consejo Audiovisual de Andalucía. Recurso en línea: http://www.consejoaudiovisualdeandalucia.es/sites/default/files/ publicaciones/estudio_sobre_gxnero_y_deporte_en_televisixn_2008. pdf [Consulta: 22 de julio de 2013].

7. Cunningham, G.B., Sagas, M., Satore, M.L., Amsden, M.L. y Schellhase, A. (2004). Gender representation in the NCAA News: Is the glass half full or half empty? Sex Roles: A Journal of Research, 50 (11/12), 861870 .

8. Duncan, M. C. (1990). Sports photographs and sexual difference: Images of men and women in the 1984 and 1988 Olympic Games. Sociology of Sport Journal, 7, 22-43.

9. Duncan, M. C. y Messner, M. A. (1994). Gender stereotyping in televised sports: A follow up to the 1989 study. Estados Unidos: The Amateur Athletic Foundation of Los Angeles. Recurso en línea http://www.la84.org/gender-stereotyping-in-televised-sports-a-followup-to-the-1989-study [Consulta: 22 de julio de 2013].

10. Duncan, M. C. y Messner, M. A. (2000). Gender in televised sports: 1989, 1993 and 1999. Estados Unidos: The Amateur Athletic Foundation of Los Angeles. Recurso en línea: http://library.la84.org/9arr/ ResearchReports/tv2000.pdf [Consulta: 22 de julio de 2013].

11. Duncan, M. C., Messner, M. A. y Williams, L. (1991). Coverage of women's sports in four daily newspapers. Estados Unidos: The Amateur Athletic Foundation of Los Angeles. Recurso en línea: http://www.la84. org/coverage-of-womens-sports-in-four-daily-newspapers [Consulta: 22 de mayo de 2012].

12. Duncan, M. C., Messner, M. A., Williams, L. y Jensen, K. (1990). Gender stereotyping in televised sports. Estados Unidos: The Amateur Athletic Foundation of Los Angeles. Recurso en línea: http://www.la84.org/gender-stereotyping-in-televised-sports/ [Consulta: 22 de julio de 2013].

13. Fink, J. y Kensicki, L. (2002). An imperceptible difference: Visual and textual constructions of femininity in Sports Illustrated and Sports
Illustrated for Women. Mass Communication and Society, 5, 317-339. Recurso en línea: http://dx.doi.org/10.1207/S15327825MCS0503_5. [Consulta: 22 de mayo de 2012].

14. Frideres, J.E. y Palao, J. Ma . (2006). Análisis de las noticias deportivas de dos periódicos digitales de Espańa y Estados Unidos: ¿promoción de la actividad física y el deporte? Apunts, 85, 7-14.

15. Kane, M.J. y Greendorfer, S.L. (1994). The media's role in accommodating and resisting stereotyped images of women in sport. En P. J. CREEDON (Ed.) Women, media and Sport: Challenging gender values (pp. 28-44). Thousand Oaks, CA: Sage Publications.

16. López Díez, P. (2011). Deporte, mujeres y medios de Comunicación. Sugerencias y recomendaciones. Madrid: Consejo Superior de Deportes.

17. Lumpkin, A.; Williams, L. D. (1991). An analysis of Sports Illustrated feature articles, 1954-1987. Sociology of Sport Journal, 8(1), 16-32.

18. MARCA. Desde 1979 - 2011

19. MUNDO DEPORTIVO. Desde 1979 - 2010

20. Pedersen, P.M. (2002). Examining equity in newspaper photographs: A content analysis of the print media photographic coverage of interscholastic athletics. International Review for the Sociology of Sport, December 2002, 37(3-4), 303-318

21. Ramajo, N. y Lallana, I. (2011). Olimpismo y los estudios de género. En E. Fernández, B. Cerezuela, M. Gómez., C. Kennett y M. de Moragas (Ed.), Mosaico Olímpico. Investigación multidisciplinar y difusión de los estudios olímpicos CEO-UAB, 20 años, (pp.181-188). Barcelona: Ayuntamiento de Barcelona y Centro de Estudios Olímpicos de la Universidad Autónoma de Barcelona.

22. Rintala, J.; Birrell, S. (1984). Fair treatment for the active female: a content analysis of Young Athlete magazine. Sociology of Sport Journal, 1(3), 231 - 250.

23. Sainz de Baranda Andújar, Clara (2013). Mujeres y deporte en los medios de comunicación. Estudio de la prensa deportiva española (1979-2010). [Tesis doctoral]. Universidad Carlos III de Madrid.

24. SPORT. Desde 1979 - 2010

25. WACC (2000). Global Media Monitoring Project: Women's Participation in the News. Toronto: Media Watch Canada.

26. WACC (2005). Promoting Gender Equality in News Media: GMMP 2005. Toronto: Media Watch Canada.

27. WACC (2010). ¿Quién figura en las noticias? Proyecto de Monitoreo Global de Medios. Toronto: Asociación Mundial para la Comunicación Cristiana (WACC). Recurso en línea: http://www.adpc.cat/05 ComFem/document/Informe\%20EspanyolGMMP_2010.pdf [Consulta: 22 de julio de 2013]. 